\title{
Evaluation of the HB\&L carbapenemase and extended spectrum beta lactamase-AmpC automated screening kits for the rapid detection of resistant Enterobacteriaceae in rectal swabs
}

\author{
Sara Marani, Maria Federica Pedna \\ Unit of Microbiology, The Greater Romagna Hub Laboratory, Pievesestina di Cesena, Italy
}

\begin{abstract}
Summary
Background. In the past two decades, a rapid increase of infections due to multidrug-resistant Enterobacteriaceae was reported worldwide, including in Italy. These bacteria express genes encoding for extended-spectrum $\beta$-lactamases (ESBL) or bear a plasmid-mediated AmpC that induce phenotypically a resistance to the last-generation cephalosporins; even more worrying is the rapid increase of Enterobacteriaceae carrying genes conferring resistance to carbapenems (CPE).

Materials and methods. The gut may serve as reservoir for these antibiotic drug-resistant bacteria: as a consequence, the rapid detection of drug resistant Enterobacteriaceae from
\end{abstract}

Correspondence: Sara Marani, Unit of Microbiology, The Greater Romagna Hub Laboratory, P.zza della Liberazione 60, Pievesestina (FC), Italy.

Tel.: +39.0547.394872/4863 - Fax: +39.0547.394801

E-mail: sara.marani4@gmail.com

Key words: Enterobacteriaceae; extended-spectrum beta-lactamase; carbapenemas; rectal swab; screening.

Acknowledgments: the authors would like to thank Alifax (Padua, Italy) for the support provided to this work.

Contributions: SM contributed to samples's collection and performed the evaluation of the Alfred 60AST and Sidecar systems, made the data analysis and wrote the manuscript; MFP supervised the work, contributed to the data analysis and to the writing of the manuscript.

Conflict of interest: Dr. Marani reports grants from Alifax (Padua, Italy), as a support to perform this study.

Received for publication: 15 March 2017.

Accepted for publication: 20 March 2017.

(C) Copyright S. Marani and M.F. Pedna, 2017

Licensee PAGEPress, Italy

Microbiologia Medica 2017; 32:6709

doi:10.4081/mm.2017.6709

This article is distributed under the terms of the Creative Commons Attribution Noncommercial License (by-nc 4.0) which permits any noncommercial use, distribution, and reproduction in any medium, provided the original author(s) and source are credited. rectal swabs is an important tool to identify rectal carriage of resistant bacteria. This procedure is the basic tool to successfully implement the infection control measures in the hospital wards. The study evaluated the capability of the HB\&L ESBL/AmpC and CARBAPENEMASE screening kit (Alifax, Padua, Italy) to rapidly identify the drug resistant enterobacteriaceae from rectal swabs: the performance was compared with the conventional method.

Results and conclusions. The overall agreement was very good $(91 \%$ for the detection of ESBL-AmpC, and $96.2 \%$ for the identification of CPE); this method is thus an efficient tool to quickly report positive multidrug resistant bacteria in rectal swabs.

\section{Introduction}

The phenomenon of multi-drug resistance is now emerging at an alarming rate among a variety of bacterial species, causing both nosocomial and community-acquired infections; members of the Enterobacteriaceae are among the mayor causative agents of nosocomial infections $(2,5)$.

One of the most important traits of these germs is the resistance to $\beta$-lactams in Gram-negative bacilli from which different classes of drug inactivating enzymes have been isolated from different bacterial species: these include chromosomally and plasmid encoded $\beta$-lactamases.

Escherichia coli and Klebsiella pneumoniae are among the most common producers of extended-spectrum betalactamases (ESBL).

The prevalence of extended spectrum $\beta$-lactamase producing Enterobacteriaceae has increased sharply over the last decade (7).

The ESBL are Amber Class A $\beta$-lactamase and may be defined as plasmid-mediated enzymes that hydrolyse Oxymino-Cephalosporins and Monobactams but not Cephamycins or Carbapenems. The activity of these enzymes is currently in vitro inhibited by clavulanic acid, sulbactam and tazobactam.

The AmpC $\beta$-lactamase are Amber Class $\mathrm{C}$ enzymes and they are typically chromosomal encoded enzyme in many Gram-negative bacteria; in contrast to the ESBL this type of $\beta$-lactamase hydrolyses a broad and extended-spec- 
trum of Cephalosporins but are not inhibited by $\beta$-lactamase inhibitors such as the clavulanic acid (4).

While the ESBL producing enterobacteriaceae carriage often persists for years without disease, the CPE can occasionally cause bloodstream and urinary tract infections even in patients without clearly identifiable healthcare-associated risk factors (3).

A more recently emerged and worrying phenomenon is the rapid increase of Carbapenemase-producing enterobacteriaceae (CPE) worldwide. Carbapenemases were able to hydrolyse most of the $\beta$-lactams drugs, including the carbapenems (imipenem, ertapenem, meropenem and doripenem); their activity is inhibited by boronic acid and, at least partially, by clavulanic acid and tazobactam (5).

The high consumption of carbapenem antibiotics associated with the co-selection by other antibiotics (e.g. cephalosporins), the large diffusion of those bacteria in the community, the uncontrolled increase and transmission in the hospital environment, that is largely owed to undiagnosed carriers, are among the main reason for their rapid spread.

Enterobacteriaceae belong to the normal human intestinal flora so that the gastrointestinal tract functions as reservoir for potentially drug resistant bacteria; the carrier patients represent the most important potential source of cross-transmission in the healthcare setting and therefore all the patients at risk (e.g. patients hospitalized in ICUs or wards with high incidence of CPE infection, contacts of newly discovered carriers, those transferred from another hospital with high incidence of CPE infection, immunocompromised patients including transplanted patients) should be screened for the CPE carrying status. The recommended samples for these screening are faecal or rectal swabs (2).

The infections caused by multidrug resistant Enterobacteriaceae are particularly difficult to treat and they are consequently associated with high mortality and morbidity. Therefore the implementation of a reliable, rapid and sensitive screening test for the rapid identification of subjects that are rectal carriers of resistant bacteria among hospitalized patients is essential for the success of the infection control measures in a timely manner before they transmission occurs (e.g. assignment of colonized or infected patients to a single room and the use of disposable gown and gloves for all the personnel that are in contact with these patient) $(1,6)$.

Different diagnostic methods are available for the identification of the antibiotic resistant germs: phenotypic and molecular-based techniques are capable to identify carbapenemase and $\beta$-lactamase producing bacteria, with variable efficiencies.

The molecular methods are highly sensitive and reliable for the early identification (they usually can give results within 4-6 hours) but are indeed still quite expensive and not readily available in many laboratories. As the emergence of the drug resistant enterobacteriaceae are increasingly reported, the detection of carriers subjects still relies mostly on the use of screening assays, based on culture media, for the initial detection of these strains. After having identified the potential resistant isolates, the resistance status must then be confirmed by using antibiotic susceptibility test (e.g. combination disk test) and/or molecular techniques like PCR and sequencing able to identify genes encoding enzyme (4).

The aim of the study was to evaluate the capability of the HB\&L ESBL/AmpC and CARBAPENEMASE screening kit to quickly identify extended spectrum $\beta$-lactamase $\mathrm{AmpC}$ and carbapenemases producing enterobacteriacae in rectal swabs from potentially colonised subjects.

The basic method is performed in automation with the use of the Alfred $60^{\mathrm{AST}}$ and Sidecar systems and consists of an enrichment growth step in broth containing the appropriate antimicrobial and antifungal supplements (so that the system could inhibit the growth of gram-positive bacteria and non$\mathrm{ESBL} / \mathrm{AmpC} / \mathrm{CPE}$ producing enterobacteriacae) for a total incubation time of $6 \mathrm{~h} 30 \mathrm{~min}$. At the end of the incubation period, all the positive samples are automatically plating on selective agar medium standard plates. This newly available method and the conventional culture, actually applied in the routine screening at Unit of Microbiology, the Greater Romagna Hub laboratory, were compared.

\section{Materials and methods}

A total of 205 E-Swabs ${ }^{\mathrm{TM}}$ (Copan, Brescia, Italy), randomly selected among those received in the Unit of Clinical Microbiology, were included in the study: 100 samples were processed with the HB\&L ESBL-AmpC screening kit with unknown result and the remaining 105 samples, choosing 51 positive and 54 negative according to routine method applied in the laboratory, were processed with HB\&L CARBAPENEMASE screening kit. Both kits were used on the Alifax microbiology line analysers (Alfred 60 AST and Sidecar systems). These instruments are based on a laser scattering reading system that automatically detect and enumerate the growth of germs into broth vials and an additional automated plates streaking tool.

For the screening of ESBL and AmpC producing enterobacteriaceae the results obtained with that automated system were compared with the conventional streaking on selective Agar ChromID ESBL (bioMèrieux, Marcy L'Etoile, France): these plates contain a mixture of antibiotics, including Cefpodoxime, and chromogenic substrates that helps rapid identification of ESBL-positive enterobacteriaceae species.

The results obtained with the HB\&L CARBAPENEMASE kit were compared with the routine method including streaking of primary samples on Chrom ID CARBA/OXA (bioMèrieux), a selective chromogenic medium containing a mixture of antibiotics which enables the selective growth of $\mathrm{CPE}$, and three chromogenic substrates which allow the visual identification of the most frequently isolated CPE strains.

Each rectal swab was be loaded directly into Alfred $60^{\mathrm{AST}}$ and Sidecar systems that automatically inoculated a fixed volume $(200 \mu \mathrm{L})$ into individual vials of the 
enrichment broth (containing $1.8 \mathrm{~mL}$ of growth medium) supplemented with an antimicrobial and antifungal solution $(200 \mu \mathrm{L})$ in order to inhibit the growth of gram positive and non-ESBL/AmpC/CPE producing enterobacteriaceae.

The vials were then incubated at $37 \pm 1^{\circ} \mathrm{C}$ for $6 \mathrm{~h}$ and 30 min, under the use of a magnetic vortex. During this time the light scattering signals are detected and displayed in real time as growth curves, and a count evaluation $(\mathrm{CFU} / \mathrm{mL})$ is obtained by a dedicated algorithmic calculation.

At the end of incubation time, subcultures $(10 \mu \mathrm{L}$ volume) of the vials automatically identified as non-negative (thus including the following three categories: positive, turbid and invalid) were plated on chromogenic and selective agar media for the strain isolation and consequent identification. The samples tested with the HB\&L ESBL/AmpC screening kit were plated on ChromID ESBL and those examined with the HB\&L CARBAPENEMASE screening kit were plated on ChromID CARBA.

At the end of the enrichment step the samples were sufficiently enriched to perform further biochemical tests from broth (e.g. oxidase test strips) and also allowed the use of phenotypic and genotypic confirmatory assays.

In detail, all the samples found positive by the HB\&L ESBL-AmpC screening method were processed with a disk diffusion test (ROSCO DIAGNOSTIC A/S, Taastrup, Denmark) to confirm the resistance phenotype. The following antibiotics were included in the confirmatory method: Cefotaxime + Cloxacillin, Ceftazidime + Cloxacillin, Cefotaxime + Clavulanate, Ceftazidime + Clavulanate, Cefotaxime + Cloxacillin + Clavulanate and Ceftazidime + Clavulanate + Cloxacillin in order to distinguish between ESBL and AmpC producing enterobacteriaceae.

As far as the evaluation of the HB\&L CARBAPENEMASE screening method is concerned, only the samples found as positive with this method and being, negative according to the routine method, were confirmed with a disk diffusion test (ROSCO DIAGNOSTIC A/S, Taastrup, Denmark) based on a combination of Meropenem, Meropenem + Phenylboronic acid, Meropenem + Cloxacillin, Meropenem + Dipicolinic acid and Temocillin.

The final bacterial identification was obtained using Vitek MS MALDI-TOF technology (bioMèrieux) on colonies isolated from automatically seeded plates.

\section{Results}

\section{HB\&L ESBL-AmpC screening kit}

Twenty-five out of the 100 evaluated samples, were turbid (and consequently not interpretable by the software), 9 were invalid because of wrong volumetric dispensation or insufficient sample volume. These 34 specimens were only evaluated based the results obtained from colonies grown in automatically seeded agar plates without taking into consideration the finding of the laser scattering reading system.

In detail 47 positive and 39 negative were detected for both the methods used. The HB\&L ESBL-AmpC screening kit identified 12 specimens that were not confirmed by the standard techniques and 2 negative specimens for this method were positive when routinely evaluated. Both of these potentially false negative for the HB\&L ESBL-AmpC test were further evaluated by using the disk diffusion method applied on fresh colonies: one was found positive for E.coli producing ESBL and finally scored as false negative, whereas the second showed the presence of P.fluorescens so not fitting within the scope of the HB\&L ESBLAmpC method and finally scored as true negative.

Among the 12 positive samples identified by the HB\&L ESBL-AmpC method and reported as negative by the standard techniques, 8 were found positive for $\beta$-lactamase producing bacteria: in particular 6 ESBL producing E.coli , 1 ESBL+AmpC producing E.coli were identified and $1 \mathrm{sam}-$ ple showed the contemporary presence of both an ESBL producing E.coli and an AmpC producing Enterobacter aerogenes.

The remaining 4 samples were negative for $\beta$-lactamase production when additionally tested for confirmation: 1 was positive for non enterobacteriaceae bacteria, 2 were positive for not resistant $K$. pneumoniae and 1 showed the presence of susceptible strain of $P$. aeruginosa, E. coli and $K$. pneumoniae. In conclusion the HB\&L ESBL-AmpC method showed 4 false positive specimens.

Table 1 summarizes the comparative results calculated after the performance of the confirmatory disk diffusion test with agreement equal to $91 \%$. In detail, the HB\&L ESBLAmpC screening method overall reported 1 false negative and 4 false positive specimens after revaluation of the discrepant results with the phenotypic confirmation.

The Cohen's kappa coefficient was calculated to evaluate the agreement among these tests after confirmation was 0.821 corresponding to an excellent agreement (among 0.81-1.00) according to the Altman guidelines.

\section{HB\&L CARBAPENEMASE screening kit}

Among 105 rectal swabs tested with the HB\&L CARBAPENEMASE screening kit, 60 were positive and 45 were negative, before applying the confirmatory method: in detail, 28 samples $(26.7 \%)$ were turbid and $2(1.9 \%)$ were invalid: as a consequence for these samples the results was considered following the bacterial growth on the agar medi-

Table 1. Comparative results obtained for the identification of ES L-AmpC producing enterobacteriaceae with the HB\&L ES LAmpC screening kit and the routine conventional method after confirmatory testing with the disk diffusion test.

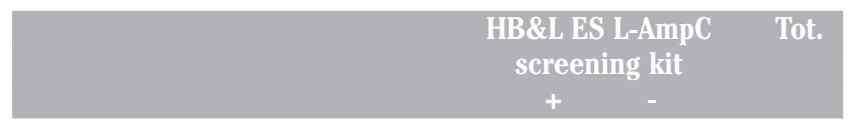

Standard method (ChromID ES L

and disk diffusion test)

\begin{tabular}{cccc}
+ & 47 & 1 & 48 \\
- & 8 & 44 & 52 \\
Tot. & 55 & 45 & 100 \\
\hline
\end{tabular}


um. It is of note that 9 negative samples according to routine method, that found only 51 positive, were identified positive by the new kit.

For this samples was performed a confirmation test based on disk diffusion on Mueller Hinton agar: 4 were colonized by KPC producing $K$. pneumoniae, 3 samples were colonized by susceptible $K$. pneumoniae, one by susceptible E.coli and one by Enterococcus faecium (Table 2).

The results obtained with the HB\&L CARBAPENEMASE screening kit agreed at $96,2 \%$ with the reference screening method plus confirmation test.

Cohen's kappa index was calculated to evaluated the agreement among the tests. The index was 0.924 corresponding to an excellent agreement (among 0.81 and 1 values) according to Altman guidelines.

\section{Discussion and Conclusions}

According to results obtained, the ESBL/AmpC screening kit is an efficient and very accurate screening method for rapidly detection of ESBL/AmpC producing strains in rectal swabs; the overall agreement was $91 \%$.

The method was able to identify 8 moreover of positive samples, that were not identified with the traditional plating on agar medium.

The kit can be an efficient support to screen $\beta$-lactamase producing bacteria reducing the spread of resistance in critical hospital setting.

According to results obtained, the HB\&L CARBAPENEMASE screening kit was able to identify carbapenemaseproducing Enterobacteriaceae from rectal swabs; it was able to identify 4 positive samples more than routine

Table 2. Comparative results obtained for the identification of carbapenemase producing enterobacteriaceae with the HB\&L CARBAPENEMASE screening kit and the routine conventional method with the disk diffusion test.

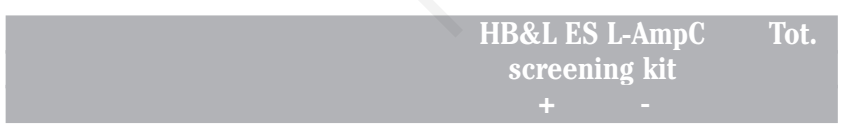

Standard method (ChromID ES CARBA/OXA L and disk diffusion test)

\begin{tabular}{cccc}
+ & 51 & 0 & 51 \\
- & 4 & 50 & 54 \\
Tot. & 55 & 50 & 105 \\
\hline
\end{tabular}

method with agreement of $96.2 \%$. The incubation in selective enrichment broths seems to be efficient to identify the resistant bacteria.

These screening methods are totally automated, from inoculation of samples into broths to plating of positive samples on selective agar media; results can be exhibit within $6 \mathrm{~h} 30 \mathrm{~min}$, and therefore they reduce the workload and optimize the laboratory workflow.

The rapid recognition of antimicrobial-resistant organisms is essential to minimize the spread of these bacteria and to apply the control measures like isolation and monitoring of colonized patients in critical hospital wards.

\section{References}

1. Adler A, Navon-Venezia S, Moran-Gilad J, et al. Laboratory and clinical evaluation of screening agar plates for detection of carbapenem-resistant Enterobacteriaceae from surveillance rectal swabs. J Clin Microbiol 2011;49:2239-42.

2. Hrabak J, Chudackova E, Papagiannitsis C. Detection of carbapenemases in Enterobacteriaceae: a challenge for diagnostic microbiological laboratories. Clin Microbiol Infect 2014;20:839-53.

3. Huttner B, Haustein T, Uckay I, et al. Decolonization of intestinal carriage of extended-spectrum- $\beta$-lactamaseproducing Enterobacteriacae with oral colistin and neomycin: a randomized, double-blind, placebo-controlled trial J Antimicrob Chemother 2014;68:2375-82.

4. Luzzaro F, Gesu G, Pagani L, Rossolini G. Diagnostica delle beta-lattamasi a spettro esteso (ESBL) nelle Enterobacteriaceae: problemi e raccomandazioni nella realtà epidemiologica italiana. Microbiol Med 2007;22: 281-90.

5. Nordmann P, Gniadkowski M, GIske CG, et al. Identification and screening of carbapenemase-producing Enterobacteriaceae. Clin Microbiol Infect 2012;18: 432-8.

6. Sing K, Magold K, Wyant K, et al. Rectal screening for Klebsiella pneumoniae carbapenemases: comparison of real-time PCR and culture using two selective screening agar plates. J Clin Microbiol 2012;50:2592-600.

7. Thenmozhi S, Moorthy K, Sureshkumar BT, Suresh M. Antibiotic resistance mechanism of ESBL producing Enterobacteriaceae in clinical field: a review. Int J Pure App Biosci 2014;12:207-26. 